\section{Moving more: our heart cares but do our lungs?}

\author{
Rachael A Evans ${ }^{1,2}$
}

Physical inactivity is the fourth leading risk factor for global mortality across low-income, middle-income and high-income countries. ${ }^{1}$ Human movement developed for endurance, yet the technological advancements of the last 50-100 years such as cars, television, computers and the internet have synergistically reduced daily activity. Evolutionary processes, usually occurring over hundreds of thousands to millions of years, have been unable to keep pace with the changing sociology. Human daily energy expenditure, previously stable over the past 3.5 million years, has reduced over the last 50 years concurrent with a dramatic increase in high energy food availability. $^{2}$

The excess mortality caused by physical inactivity is largely secondary to coronary heart disease (CHD) and other cardiovascular diseases (CVD). A doseresponse relationship is apparent whereby 'moderate' physical activity is associated with a $20 \%-25 \%$ risk reduction in either CHD or CVD compared with a 30\%-35\% risk reduction for 'high' physical activity. ${ }^{3}$ Current WHO recommendations for physical activity reflect these statistics advising either at least $150 \mathrm{~min}$ of moderate physical activity per week or $75 \mathrm{~min}$ of vigorous physical activity and also highlight increased benefit of either 300 min of moderate weekly physical activity or $150 \mathrm{~min}$ of vigorous physical activity. Moderate physical activity is typically described around the level of brisk walking whereas running would be categorised as vigorous physical activity. In high-income countries, at least 25\%-35\% of the population do not achieve these recommendations.

In Thorax, Fuertes and colleagues ${ }^{4}$ investigate whether levels of physical activity or changes in physical activity are associated with better lung function using data collected from phases II and III of the European Community Respiratory Health

${ }^{1}$ Infection, Inflammation and Immunity, University of Leicester, Leicester, UK

${ }^{2}$ Glenfield Hospital, NIHR Leicester BRC (Respiratory), Leicester, UK

Correspondence to Rachael A Evans, Glenfield Hospital, University Hospitals of Leicester NHS trust, University of Leicester, Leicester LE3 9QP, UK; re66@le.ac.uk
Study (ECRHS). They compared spirometric data with vigorous physical activity levels via a self-report questionnaire at two time points approximately 10 years apart in 3912 predominantly young to middleaged adults. Higher weekly frequency and duration of vigorous physical activity was associated with better lung function over time in smokers, but not in non-smokers. There was no association between vigorous activity levels and lung function decline in either group. Changing physical activity status from 'non-active' to 'active' was associated with higher $\mathrm{FEV}_{1}$ and FVC at follow-up.

As with any type of study, careful yet respectful interpretation of epidemiologically derived associations is needed leading to experiments investigating precise causal mechanisms. For example, the pioneering study by Doll and Hill demonstrated an association between smoking and development of lung carcinoma $\mathrm{a}^{5}$ and subsequent studies described the carcinogen. Understanding whether there is a true association in population studies requires consideration of measurement error, biases and confounding counterbalanced by the large population sizes typically involved. Fuertes and colleagues attempted to reduce selection bias, adjusted for confounding where possible and used longitudinal data for both measurements. They sought to reduce reverse causation by separately studying individuals prior to disease or symptom occurrence. Their data support a previously reported association between high physical activity levels and reduced lung function decline similarly apparent in smokers but not in non-smokers or ex-smokers, ${ }^{6}$ and another recent report where remaining active, becoming active or being active at baseline but then becoming inactive were all associated with better lung function compared with continuously being inactive. ${ }^{7}$ However, in the latter study, the difference between smokers and non-smokers was not seen and reverse causation cannot be excluded.

Spirometry may not detect early changes in the small airways and in the current study, there were issues with different spirometers being used for the first examination. However, it is unlikely that the latter would have made wholescale changes to the results and the authors also performed sensitivity analyses. As the authors discuss, a negative aspect of spirometry is the influence of changes in body mass index (BMI) which may be altered by changes in physical activity levels. However, the results in the current study were unchanged after stratification for BMI.

Daily physical activity is a variable behaviour and therefore measurement and interpretation is challenging; consider your own activity over the last week, month, year, decade-in binary form, are you active or not? 'Physical activity' has different components, for example, the duration, frequency and intensity of activity, and also the duration of stationary or sedentary time with all the accompanying computations. A range of measurements can be made from subjective, using self-report questionnaires, to objective by wearable technology such as simple pedometer derived daily step counts to more sophisticated commercially available accelerometers enabling calculation of duration and intensity of movement. In research studies, the latter are typically measured over a week, whereas questionnaires can be assessed over much larger periods of time; the balance of signal-to-noise ratio is not straightforward for either measure. With the increasing use of Self-Monitoring, Analysis and Reporting Technology (SMART) technology by the general public, potentially more objective data over prolonged periods is likely to be available (consent permitting). For the current study, weekly frequency and duration of vigorous activity described as 'exercised so much that they got out of breath or sweaty' was assessed over an undefined period of time.

When does intense physical activity become exercise? Exercise defined by the Oxford English Dictionary is an 'activity requiring physical effort, carried out to sustain or improve health and fitness'. Vigorous physical activity described in the current study was probably 'exercise' and would likely be associated with cardiorespiratory fitness for which a laboratory cardiopulmonary exercise tests can provide a well-validated objective assessment. In the current study, no relationship was seen in non-smokers between vigorous activity and lung health. However, a positive relationship between sustaining higher and relatively increased levels of cardiorespiratory fitness assessed by a maximal treadmill test and preserved lung health over 20 years was reported from the Coronary Artery Risk Development in Young Adults study 'CARDIA' cohort $(n=3332)$ in both smokers and non-smokers. ${ }^{8}$ A recent report in children and young adults showed a positive relationship in cross-sectional data between cardiorespiratory fitness, assessed by an incremental cycle ergometer test, and both 
$\mathrm{FEV}_{1}$ and FVC. ${ }^{9}$ Longitudinal data from the same report demonstrated that fitness was associated with preserved lung function supporting the results of the CARDIA study but only before peak adult lung function had been attained. ${ }^{9}$

To date, most of the data reporting a positive association between physical activity levels and a lower cardiovascular risk refer to higher intensity activity. However, sedentary behaviour has also been linked to worse cardiometabolic health. Skeletal muscle inactivity leads to insulin resistance through oxidative inflammatory pathways ${ }^{10}$ and even short interrupted breaks in sedentary behaviour can attenuate postprandial glucose and insulin levels: markers of future risk of diabetes and CVD. ${ }^{11}$ It has been postulated that physical inactivity perhaps has a causative role in COPD rather than purely being a consequence of airways disease. ${ }^{12}$ The incidence of COPD was not an outcome in the current study due to a low number of cases, yet at least $50 \%$ of the population were inactive and 25\% were smokers. Impairments in metabolic health such as diabetes and metabolic syndrome appear to negatively affect lung health ${ }^{9}$ and ongoing research is being conducted to understand whether physical inactivity or sedentary behaviour causing skeletal muscle inactivity is a potential mediator.

The current study and others demonstrate an association between better lung health and higher physical activity levels only in smokers perhaps implicating the role of systemic inflammation. Individuals who are physically active have lower levels of systemic inflammation than their less active peers, ${ }^{13}$ but the precise mechanisms remain under investigation. Systemic inflammation was highlighted as a potential mechanism for the association between physical fitness and lung health described in the CARDIA cohort as both C-reactive protein and fibrinogen levels were also indirectly associated. ${ }^{6}$ However, if systemic inflammatory pathways were the predominant link, perhaps exercise training in airways diseases such as asthma and COPD might also be expected to lead to improvements in lung function via similar mechanisms, yet this is not described. The mechanisms of benefit from pulmonary rehabilitation are known to occur through the systemic features of lung diseases rather than alterations in pulmonary function. ${ }^{14}$ A meta-analysis involving individuals with asthma also showed no alteration in lung function with exercise training, ${ }^{15}$ although a longitudinal study demonstrated a positive association between physical activity levels and lung function. ${ }^{16}$ Overall, a biological explanation is needed to understand the potential links between physical activity levels, cardiorespiratory fitness, systemic inflammation and future lung function.

In summary, what are the public health messages? The current physical activity recommendations are based on lowering cardiovascular risk to improve longevity: 'our heart cares'. As a higher risk group, alongside smoking cessation it would seem prudent to engage smokers to increase their physical activity levels known to be reduced compared with the average population; there may be longer-term pulmonary benefits but the mechanisms need to be elucidated before firm recommendations can be made on this basis. Once peak lung function has been attained in healthy adult non-smokers, it is unclear whether or how physical activity levels affect future lung function. The evidence for the promotion of physical activity and exercise for future lung health is currently strongest for children and young adults, and perhaps those with asthma should be a particular focus where the culture may be to avoid exercise for fear of worsening symptoms.

Our lungs care about moving more but youth, physical fitness and smoking may make our lungs care more.

Funding Dr Rachael Evans holds a NIHR Clinician Scientist Award CS-2016-16-020.

Disclaimer The views expressed in this article are those of the author and not necessarily those of the NHS, the NIHR, or the Department of Health and Social Care.

Competing interests None declared.

Patient consent Not required.

Provenance and peer review Commissioned; internally peer reviewed.

(C) Article author(s) (or their employer(s) unless otherwise stated in the text of the article) 2018. All rights reserved. No commercial use is permitted unless otherwise expressly granted.

\section{Check for updates}

To cite Evans RA. Thorax 2018;73:501-502.
Accepted 26 March 2018

Published Online First 16 April 2018

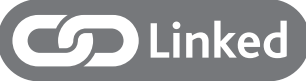

http://dx.doi.org/10.1136/thoraxjnl-2017-210947

Thorax 2018;73:501-502.

doi:10.1136/thoraxjnl-2017-211428

\section{REFERENCES}

1 World Health Organisation. Global recommendations on physical activity for health. Geneva: World Health Organisation, 2010. (accessed 7 Jan 2018).

2 Leonard WR. Size counts: evolutionary perspectives on physical activity and body size from early hominids to modern humans. J Phys Act Health 2010;7:\$284-98.

3 Shiroma EJ, Lee IM. Physical activity and cardiovascular health: lessons learned from epidemiological studies across age, gender, and race/ethnicity. Circulation 2010:122:743-52.

4 Fuertes E, Carsin AE, Antó JM, et al. Leisure-time vigorous physical activity is associated with better lung function: the prospective ECRHS study. Thorax 2018:73:376-84.

5 Doll R, Hill AB. Smoking and carcinoma of the lung; preliminary report. Br Med J 1950;2:739-48.

6 Garcia-Aymerich J, Lange P, Benet M, et al. Regular physical activity modifies smoking-related lung function decline and reduces risk of chronic obstructive pulmonary disease: a population-based cohort study. Am J Respir Crit Care Med 2007;175:458-63.

7 O'Donovan G, Hamer M. The association between leisure-time physical activity and lung function in older adults: The English longitudinal study of ageing. Prev Med 2018;106:145-9.

8 Benck LR, Cuttica MJ, Colangelo LA, et al. Association between cardiorespiratory fitness and lung health from young adulthood to middle age. Am I Respir Crit Care Med 2017;195:1236-43.

9 Hancox RJ, Rasmussen F. Does physical fitness enhance lung function in children and young adults? Eur Respir J 2018;51:17.

10 Gratas-Delamarche A, Derbré F, Vincent S, et al. Physical inactivity, insulin resistance, and the oxidativeinflammatory loop. Free Radic Res 2014;48:93-108.

11 Henson J, Davies MJ, Bodicoat DH, et al. Breaking up prolonged sitting with standing or walking attenuates the postprandial metabolic response in postmenopausal women: a randomized acute study. Diabetes Care 2016:39:130-8.

12 Hopkinson NS, Polkey MI. Does physical inactivity cause chronic obstructive pulmonary disease? Clin Sci 2010;118:565-72.

13 Albert MA, Glynn RJ, Ridker PM. Effect of physical activity on serum C-reactive protein. Am J Cardiol 2004;93:221-5

14 Spruit MA, Singh SJ, Garvey C, et al. An official American Thoracic Society/European Respiratory Society statement: key concepts and advances in pulmonary rehabilitation. Am I Respir Crit Care Med 2013;188:e13-e64.

15 Carson KV, Chandratilleke MG, Picot J, et al. Physical training for asthma. Cochrane Database Syst Rev 2013:9:CD001116.

16 Brumpton BM, Langhammer A, Ferreira MA, et al. Physical activity and incident asthma in adults: the HUNT Study, Norway. BMJ Open 2016;6:e013856. 